\title{
ATP, an Extracellular Signaling Molecule in Red Blood Cells: A Messenger for Malaria?
}

\author{
Ghania Ramdani, Gordon Langsley
}

\begin{abstract}
Adenosine $5^{\prime}$ triphosphate (ATP), discovered in 1929 by Karl Lohmannest, is described as an essential energy source for cells. In the biochemistry of all living organisms, ATP hydrolysis provides the energy required for the chemical reactions of metabolism. It is the precursor of a number of essential enzyme cofactors, such as nicotinamide adenine dinucleotide $\left(\mathrm{NAD}^{+}\right)$ and coenzyme A [NAD ${ }^{+}$, flavin adenine dinucleotide (FAD), and is ATP coenzyme A are all formed from ATP] and is the source of the phosphoryl group in most kinase-mediated phosphorylation reactions. Another essential, but less known function is that ATP plays a very important role as an extracellular signaling molecule, allowing cells and tissues to communicate. ATP is converted into cAMP, a major second messenger involved in many cellular processes, by adenylyl cyclase, a membrane-associated enzyme. In this review, we describe the role of ATP as a beneficial extracellular molecule released by healthy red blood cells (RBCs) in response to hypoxia

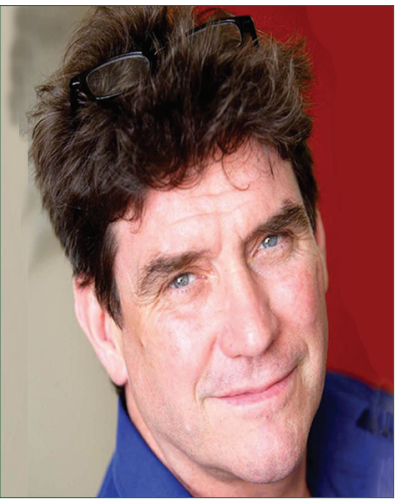

Dr. Gordon Langsley to mediate a vasodilator signal, by oxidatively stressed RBCs, and by Plasmodium falciparum-infected RBCs (iRBCs), and its similarity with released ATP that by the combined action of the ectonucleotidases CD39 and CD73 is converted to adenosine that mediates sickling in sickle cell disease (SCD). (Biomed J 2014;37:284-292)
\end{abstract}

Key words: adenosine, adenosine $5^{\prime}$ triphosphate, CD39, CD73, malaria, Plasmodium, red blood cell

\section{Adenosine 5' triphosphate released from RBCs}

\section{Adenosine $5^{\prime}$ triphosphate as a vasodilator signal}

$\mathrm{R}_{\mathrm{H}}^{\mathrm{B}}$ BCs mainly facilitate oxygen transport in the body. However, this is not the only function of these abundant enucleated cells, whose cytoplasm is rich in hemoglobin and poor in organelles; they are also redoubtable sensors of oxygen allowing local vascular control. ${ }^{[1]}$

In 1992, Bergfeld and Forrester reported that human RBCs release adenosine $5^{\prime}$ triphosphate (ATP) in response to the combined effects of hypoxia and hypercapnia (abnormally elevated levels of blood carbon dioxide) ${ }^{[2]}$ More recent studies show that exposure to reduced oxygen tension $(\sim 35 \mathrm{mM} \mathrm{Hg})$ is sufficient to stimulate ATP release from the RBCs of hamsters, ${ }^{[3]}$ rabbits ${ }^{[4]}$ rats, ${ }^{[5]}$ and humans. ${ }^{[6]}$ Indeed, ATP is released from RBCs in blood samples subjected to hypoxia. ${ }^{[7]}$
In addition to RBCs, it is known that endothelial cells also release ATP when they are exposed to a high shear stress. ${ }^{[8]}$

Decrease in oxygen pressure $\left(\mathrm{pO}_{2}\right.$ : partial pressure of oxygen, that is the amount of oxygen in the blood, normally referred to as dissolved oxygen in blood) is caused by an increase of cellular oxygen consumption or a decrease in oxygen supply or systemic hypoxia, all of which can trigger RBC vasodilator signals to compensate for the mismatch between the supply and demand of oxygen ${ }^{[7]}$ Lack of $\mathrm{O}_{2}$ triggers RBC deoxygenation and induces a cascade of events including nitric oxide (NO) production by deoxyhemoglobin, mediated by nitrite reduction, i.e. the reaction between nitrite and deoxygenated hemoglobin provides a mechanism by which NO is synthesized in vivo. ${ }^{[9]}$ This leads to the possible formation of $S$-nitrosohemoglobin $(\mathrm{SNO}-\mathrm{Hb}) \cdot{ }^{[7]}$ $\mathrm{SNO}-\mathrm{Hb}$ is the bioactive form of $\mathrm{NO}$ in blood and plays an important role in regulating the blood flow. ${ }^{[10]}$ ATP is

From the Laboratory of Comparative Cell Biology of Apicomplexa, Department of Immunology, Inflammation and Infection, Cochin Institute, INSERM U1016, CNRS UMR 8104, University Paris-Descartes, Paris, France

Received: Dec. 09, 2013; Accepted: Apr. 08, 2014

Correspondence to: Dr. Gordon Langsley, Cochin Institute, INSERM U1016, CNRS UMR 8104, University Paris-Descartes, Paris, France. 27, rue du Faubourg-Saint-Jacques, 75014 Paris, France. Tel: 33-140516592; Fax: 33-140516570; E-mail: gordon.langsley@inserm.fr

DOI: $10.4103 / 2319-4170.132910$ 
released and contributes to the regulation of blood flow and $\mathrm{O}_{2}$ delivery to tissues to satisfy the metabolic needs of cells. ${ }^{[5,10]}$ ATP is released upon activation of a signaling pathway that is triggered when RBCs enter an area in which the oxygen demands of tissues exceed supply. Thus ATP acts in a paracrine manner to increase vascular caliber leading to an increase in oxygen supply.

\section{How $R B C$ s release ATP}

ATP efflux is linearly related to hemoglobin saturation with oxygen $;{ }^{[5]}$ the more saturated the hemoglobin is, the less ATP is released, suggesting that the conformational change in hemoglobin and its desaturation when the oxygen levels drop causes ATP release. Thus, physiological and pharmacological stimuli induce ATP release from RBCs. Physiologically, RBCs release ATP in response to mechanical deformation ${ }^{[11]}$ that occurs as RBCs flow throughout the body, from arteries to veins and capillaries, as well as passing through the slits in the spleen, ${ }^{[12]}$ and in response to reduced oxygen tension. ${ }^{[2]}$ Further, RBCs can release ATP in a dose-dependent manner following activation by $\beta$-adrenergic receptors present at the RBCs' surface or via prostacyclin receptors. ${ }^{[13]}$

In recent years, Sprague's group described a pathway for ATP release from RBCs. This pathway includes the heterotrimeric $\mathrm{G}$ proteins $\mathrm{Gs}$ and $\mathrm{Gi},{ }^{[13-15]}$ adenylyl cyclase $(\mathrm{AC}),{ }^{[16]}$ protein kinase $\mathrm{A},{ }^{[16]}$ and cystic fibrosis transmembrane conductance regulator (CFTR) ${ }^{[17]}$ [Figure 1]. Studies have shown that activation of Gs-coupled receptors, $\beta$-adrenergic and prostacyclin (also called prostaglandin I2 or PGI2) receptors on the RBCs' surface leads to increased cAMP levels and ATP release. ${ }^{[11,13]}$ Gs is the AC-activating pro-



Figure 1: $P$. falciparum-infected RBCs secrete metabolite(s) that contribute to parasite infectivity and changes in RBC deformability. The metabolite can be ATP, which signals via P2Y receptors expressed at RBCs' surface, or an ATP-derived metabolite such as adenosine that signals via ADORA2B receptors. $\beta$-adrenergic receptors are also implicated in parasite infectivity. All these receptors are coupled to $\mathrm{G}$ proteins and are responsible for cAMP production via activating adenylyl cyclases, which convert ATP to cAMP. cAMP activates PKA that phosphorylates RBC cytoskeletal proteins resulting in a change plasma membrane properties. tein, while Gi is traditionally described as inhibiting the activity of certain isoforms of $\mathrm{AC},{ }^{[18-22]}$ but it also activates isoforms II, IV, and VII of AC that are expressed on RBCs' surface. ${ }^{[19,22,23]}$ For example, direct activation of Gi induces an increase in cAMP and ATP release by rabbit and human RBCs. ${ }^{[13,14]}$ In addition, direct activation of Gi with wasp venom extract (mastoparan) stimulates cAMP levels and, thus, ATP release from rabbit RBCs. ${ }^{[14,24]}$

\section{How ATP triggers vasodilation}

The balance between the supply and demand of $\mathrm{O}_{2}$ in metabolically active tissues is a fundamental physiological process, and RBCs play a key role in tissue oxygen supply by the controlled release of ATP in areas where $\mathrm{O}_{2}$ needs increase, by balancing microvascular $\mathrm{O}_{2}$ supply with demand in local tissues.

Indeed, RBCs contain millimolar (mM) amounts of ATP produced mainly by glycolysis. ${ }^{[1]}$ This ATP is released in response to hypoxia to trigger vasodilator signals. ${ }^{[2]}$ When ATP is released into the vascular lumen, it can interact with P2-type purinergic receptors present on the endothelium, which elicits an endothelium-dependent vasoactive response. There are two different classes of $\mathrm{P} 2$ receptors, $\mathrm{P} 2 \mathrm{X}$ and $\mathrm{P} 2 \mathrm{Y}$, and in mammals, there is a family of seven $\mathrm{P} 2 \mathrm{X}$ (P2X1-7) receptors and eight P2Y (P2Y1, P2Y2, P2Y4, P2Y6, P2Y11-14) receptors. ${ }^{[25,26]}$

Activation of purinergic receptors stimulates the synthesis and release of several vasodilators such as NO and arachidonic acid from the endothelial cells. In cerebral circulation, ATP and its related degradation products, including adenosine, are vasoactive mediators. ${ }^{[27,28]}$ For example, Forrester et al. noted that interluminal ATP was a potent vasodilator in baboon cerebral circulation. ${ }^{[29]}$ It is likely that ATP is the primary agonist and at equimolar concentrations, ADP is slightly less efficient than ATP in causing local dilatory responses and conductive responses. ${ }^{[28]}$

\section{How ATP release by RBCs is regulated}

The release of ATP by RBCs is controlled by a negative feedback mechanism. In an intact microcirculation, when $\mathrm{RBCs}$ are in contact with an NO-rich environment, NO may act as a negative feedback to inhibit release of more ATP. Incubation of human ${ }^{[11]}$ and rabbit $\mathrm{RBCs}^{[24,30]}$ with an NO donor such as spermine NONOate (used to produce a controlled release of NO in solution) prevents ATP release in response to reduced $\mathrm{O}_{2}$ tension. In addition to stimulating soluble guanylate cyclase, NO can also target either the adenylyl cyclase itself to inhibit enzyme activation ${ }^{[31,32]}$ or Gi subtypes of G proteins, ${ }^{[33]}$ both components of signal transduction pathways used for ATP release by RBCs. Thus, NO is capable of inhibiting ATP release in response to exposure to reduced oxygen tension through Gi inactivation. 


\section{Dynamics of ATP release from RBCs}

The function of extracellular ATP is very well described for RBCs that regulate blood pressure through ATP release, which acts as signaling molecule in a vasodilatory response. We have described above that RBCs can release ATP following mechanical deformation. We know now that retraction of the actin-spectrin cytoskeleton network triggers mechanosensitive ATP release and shear-dependent membrane viscosity controls the release rate. ${ }^{[34]}$

$\mathrm{RBC}$ deformation is an important trigger of ATP release. Adding chemicals to stiffen RBC cytoskeleton (e.g. diamide) decreases the amount of ATP released, suggesting that cell deformation is a necessary trigger element. ${ }^{[35]} \mathrm{RBCs}$ that play the role of oxygen carriers are susceptible to deform. To avoid abusive ATP release, the mechano-dependent ATP release mechanism should be highly regulated, and these mechanisms of RBCs mechanosensing are still controversial. Regulation occurs between the initiation of the increase in shear stress and ATP release. Even if RBCs deform significantly by short-term constrictions, no measurable ATP is released, ${ }^{[34]}$ and the amount of released ATP is dependent on the duration of the shear stress. Using microfluidic ${ }^{[35,36]}$ and microbore tubing channels, ${ }^{[37,38]}$ it was shown that the amount of ATP released is also controlled by shear stress amplitude. In summary, a large deformation of RBCs is, in itself, insufficient to trigger a significant amount of ATP release. The required condition is that RBC deformations must occur in a well-defined time scale and should be of relatively long duration to induce significant ATP release.

\section{$A T P$ release from oxidative stressed $R B C s$}

Oxidative stress reflects an imbalance between production of reactive oxygen species (ROS) and the ability of biological systems to detoxify or to repair the damage caused by these intermediates. Oxidative stress is associated with increased production of oxidized species, or a significant decrease in the effectiveness of the antioxidant defense systems such as glutathione. ${ }^{[39]}$ Disruption of normal cell redox state can cause toxic effects through production of peroxides and free radicals that cause damage to all cellular components, including proteins, lipids, and DNA. In addition, some ROS act as messengers in cellular redox signaling. Thus, oxidative stress can cause disruptions in normal cell signaling. In humans, oxidative stress may be involved in cancers, Parkinson's disease, Alzheimer's disease, and when it affects RBCs it can be responsible for sickle cell disease (SCD)..$^{[40]}$

ATP output coming from RBC deformation is clearly linked to the antioxidant defense status of cells. First, intracellular ATP levels are decreased by hydrogen peroxide $\left(\mathrm{H}_{2} \mathrm{O}_{2}\right){ }^{[41]}$ In addition to oxidative stress-caused damage and loss of glutathione, ATP levels drop due to inhibition of glyceraldehyde-3-phosphate dehydrogenase. ${ }^{[41]}$ This ATP depletion prevents controlled cell death by apoptosis and causes the cell to simply fall apart. ${ }^{[41,42]}$ So, cellular ATP is an important determinant of cell death; cells remain alive when a certain level of ATP is maintained, but when ATP falls below this level, apoptosis is activated and a severe drop in cellular ATP causes cell necrosis. ${ }^{[41]}$

In addition to the decrease of intracellular ATP levels, oxidative stress is directly correlated to ATP release. Lower levels of oxidative stress generated by treatment with dehydroepiandrosterone (DHEA, an antioxidant) lead to a decrease in nicotinamide adenine dinucleotide phosphate (NADPH) levels and decreased ATP release. ${ }^{[43]}$ NADPH acts as a glutathione reductase (GSHred) substrate in order to maintain antioxidant GSH in its reduced form, and decreased NADPH levels directly correlate with GSH production. In its reduced form, this small tripeptide is the main non-enzymatic antioxidant in RBCs. ${ }^{[44]}$ GSH is oxidized to its dimeric form GSSG to protect the cells against oxidative attack. GSH is generated at the expense of NADPH. To generate NADPH, unlike other cells that have several methods to produce it, RBCs rely solely on glucose-6-phosphate dehydrogenase (G6PD), a key enzyme of pentose phosphate pathway that oxidizes NADPH and converts glucose-6-phosphate (G6P) to 6 phosphogluconate (6GP). G6PD also serves to determine RBC age $^{[45,46]}$ and, in addition to $\mathrm{GSH}$, is decisive in the antioxidant status of RBCs. ${ }^{[47]}$ G6PD-deficient individuals are subjected to high oxidative stress due to a lack in NADPH production, and thus have a weakened antioxidant defense system. ${ }^{[43]}$ For example, G6PD activity is low in the RBCs of diabetic patients compared to healthy individuals, ${ }^{[48]}$ leading to decreased NADPH levels in these patients, making their RBCs less deformable than healthy subjects. ${ }^{[49]}$ In addition, mutations in G6PD afford protection against malaria, and for this reason, hereditary G6PD deficiency is frequent in malaria-endemic areas. ${ }^{[50]}$ The protection afforded by mutant G6PD can be due to reduced parasite invasion and/or poor parasite replication within G6PD RBCs. ${ }^{[51]}$ So, there is a direct relationship between NADPH concentrations and ATP release resulting from RBC deformation. Correlating with a decrease in NADPH concentrations, there is a decrease in ATP release stemming from RBC deformation. RBCs obtained from patients with type 2 diabetes show the same pattern ${ }^{[43]}$ confirming that by affecting deformability of RBCs, oxidative stress will lead to a decrease in the level of ATP release.

\section{ATP release following Plasmodium falciparum infection}

\section{Role of new permeation pathways - NPP}

The clinical signs of malaria are the result of the invasion and development of $P$. falciparum within RBCs. Infection is accompanied by major changes in the host red 
blood cell ${ }^{[52]}$ that affect lipid composition, deformability, and also its osmotic and transport properties. This results in sequestration of P. falciparum-infected RBCs (iRBCs), and rigidity of the host RBC leads to cerebral malaria, the most severe complication of malaria. ${ }^{[52,53]}$

During its intracellular development, P. falciparum makes the RBC appear older; iRBCs become less dense and less deformable. iRBCs lose their typical biconcave shape, take on a more spherical shape, become covered with dense microscopic projections (knobs), and expose more phosphatidylserines (PS) at their surface. ${ }^{[54]}$ iRBCs become osmotically fragile and more permeable to a wide variety of molecules through the appearance of NPP. ${ }^{[54]}$ iRBCs lose several cellular components such as sialic acid, reduced glutathione, and especially ATP [Figure 1]. The decreased levels of antioxidants and ATP have several consequences, such as clustering of the band-3 anion transporter, as well as exposure of neo-antigens that contribute to antigenic variation and increased $\mathrm{RBC}$ adhesion via PS and band-3 that allows the parasitized RBC to escape destruction by spleen. ${ }^{[54]}$

Of all the leached molecules, we will focus on ATP. Both iRBCs and, as we described above, damaged cells or cells responding to a hypoxic signal can release ATP, and its concentration is therefore increased in the culture medium. ${ }^{[5]}$ The released ATP appears to play a key role at several levels. It plays a role in infection, as parasites are unable to infect RBCs that have been depleted of their ATP. ${ }^{[5]}$ ATP also acts as an important extracellular signal to modulate the interaction between RBCs and Plasmodium, and it has been also reported to increase the intracellular $\mathrm{Ca}^{2+}$ levels within P. falciparum, P. berghei, and P. yoelii parasites, suggesting the potential presence of purinergic receptors on the surface of the parasite. ${ }^{[56]}$ ATP is also involved in the growth of $P$. falciparum and P. berghei, and treatment with suramin, a specific purinergic receptor antagonist, reduces parasite growth in vitro and in vivo, respectively ${ }^{[57]}$ Finally, ATP is involved in endothelial cell inflammation induced following infection with Plasmodium. ${ }^{[58]}$ Like oxidative stress, RBC infection by $P$. falciparum induces increased permeability of anions and organic osmolytes. Induction of osmolyte permeability in iRBCs involves autocrine purino-receptor signaling, as extracellular ATP does not induce permeability in uninfected or unoxidized RBCs. ${ }^{[57]}$

RBCs express P2Y1 receptors, and RBCs of P2Y1-deficient mice show a delayed onset of osmolyte permeability following infection by $P$. berghei or following oxidation, when compared to wild-type RBCs. ${ }^{[59]}$ As stated, iRBCs have NPP, ${ }^{[99,60]}$ and NPP first appear between 10 and $20 \mathrm{~h}$ and are maximal around $40 \mathrm{~h}$ post-invasion. ${ }^{[57]}$ At the trophozoite stage (circa $20 \mathrm{~h}$ post-infection), the iRBC membrane becomes permeable to substances such as sorbitol and glucose, ${ }^{[61]}$ and a wide variety of solutes including anions, carbohydrates, amino acids, nucleosides, and organic or inorganic cations. ${ }^{[59]}$

NPP provide the parasite with the nutrients required for rapid growth and allows the parasite to eliminate metabolic waste products. Pharmacological inhibition of NPP leads to death of parasites cultured in vitro. Thus, the parasite must obtain purine bases and pantothenic acid from the extracellular medium, ${ }^{[62]}$ and release amino acids stemming essentially from hemoglobin degradation ${ }^{[63]}$ and excrete lactic acid produced by parasite glycolysis, whose activity is 100 times more intensive than RBC glycolysis. ${ }^{[64]}$ NPP are believed to consist of two types of channel: The first is present in small numbers and is load- and size-selective, while the other one is about 100 times more abundant and is anion selective, but does not admit non-electrolytes except perhaps nucleosides. ${ }^{[60]}$

\section{Role of cAMP/protein kinase A pathway}

ATP plays an important role in P. falciparum infection. RBCs release ATP in response to parasitic infection. ${ }^{[65]}$ In a model of mouse malaria using $P$. chabaudi-infected RBCs, the released ATP acts via an autocrine loop to stimulate parasite growth, since it can bind to $\mathrm{P} 2 \mathrm{Y}$ receptors on the surface of the parasite to maintain the infection process ${ }^{[66]}$ [Figure 1]. Then the released ATP has a deleterious effect on the host, as it augments infection via its binding to P2Y expressed either on the RBCs or on the surface of the parasite. ${ }^{[6]]}$

We have described above the involvement of NPP in the release of ATP by iRBCs. Also, the contribution of hypoxia is not excluded, since iRBC adhesion to vascular endothelium creates hypoxia, which can induce ATP release by RBCs. Another hypothesis is that oxidative stress induced by $P$. falciparum infection can promote ATP release from RBCs.

In the next section, we will describe the involvement of a cAMP-dependent protein kinase A (PKA) pathway in ATP release from RBCs, which therefore implies the involvement of Gs signaling to activate AC, a key enzyme in cAMP production. Indeed, it has been shown that receptor-mediated activation of the heterotrimeric G protein Gs results in ATP release from RBCs. ${ }^{[13]}$

\section{Role of CFTR}

CFTR is an ion channel of ATP-binding cassette (ABC) transporter class, encoded by the CFTR gene. In epithelial cells, it carries chloride ${ }^{[67]}$ and thiocyanate ${ }^{[68]}$ ions across the plasma membrane. Mutations in the CFTR gene affect the chloride ion channel transport in cell membranes and leads to cystic fibrosis.

The involvement of CFTR in ATP release from RBCs has already been demonstrated. Indeed, activation of CFTR is involved in ATP release induced by mechanical deformation of rabbit and human RBCs. ${ }^{[13]} \mathrm{A}$ cAMP-dependent 
PKA pathway plays an essential role in CFTR activation, as increases in cAMP generated by $\mathrm{AC},{ }^{[69]}$ whose activity is regulated by heterotrimeric $\mathrm{G}$ proteins, ${ }^{[21]}$ induces channel activation via cAMP-dependent PKA-mediated phosphorylation of the R domain of CFTR. ${ }^{[69]}$

\section{Role of $\beta$-adrenergic receptors}

$\beta$-adrenergic receptor and adenosine receptor agonists (isoproterenol and $5^{\prime}$ - $N$-ethylcarboxamidoadenosine, respectively) stimulated parasite entry into RBCs, while the respective antagonists (propranolol and 8-(p-sulfophenyl) theophilline) inhibited entry. ${ }^{[70]}$ Heterotrimeric $G$ proteins are involved in the signal transduction pathway that leads to ATP release from the RBCs, as it has been shown that the $\beta$-adrenergic receptors coupled to $G$ proteins play an essential role in the release process. ${ }^{[13]}$ Indeed, incubation of RBCs with epinephrine or iloprost, which activate stimulatory Gs subunits via a $\beta$-adrenergic-mediated mechanism, stimulates ATP release, whereas pre-treatment with propranolol, a receptor antagonist, inhibits ATP release. ${ }^{[13]}$ The ATP released by iRBCs that enhances parasite infectivity may be derived from the activation of the $\beta$-adrenergic receptors, as it has been demonstrated that signaling via the $\beta 2$-adrenergic receptor provokes $G$ protein-regulated entry of the human malaria parasite. ${ }^{[71]}$ Also, agonists that stimulate cAMP production led to an increase in infection that could be blocked by specific receptor antagonists. ${ }^{[13]}$ Moreover, peptides that inhibit $\mathrm{G}$ protein function reduced parasite growth in vitro, ${ }^{[71]}$ and $\beta$-antagonists also reduced $P$. berghei growth in vivo. Thus, signaling via the RBC $\beta 2$-adrenergic receptor and Gos may regulate infection across different plasmodial species. As RBC G protein signaling also contributes to parasite proliferation, it may present a novel antimalarial target. ${ }^{[70]}$

\section{Modulation of membrane properties by PKA}

It has been shown previously that mechanical deformation of RBCs could stimulate the activity of the heterotrimeric $\mathrm{G}$ protein $\mathrm{Gs}$, initiating a signal transduction pathway that mediates ATP release from RBCs. ${ }^{[13]}$ Also, Zhang et al. have shown that in sickle cell RBCs, adenosine signals through ADORA2B to increase the levels of cAMP and activate PKA.$^{[72]}$ Activation of the PKA-signaling pathway leads to induction of extracellular signal-regulated kinase (ERK) 1/2 and their combined phosphorylation of adducins, dematins, and the intercellular adhesion molecule 4 (ICAM-4) $)^{[73]}$ [Figure 1]. Indeed, in mature RBCs, phosphorylation plays an important role in the regulation of membrane functions in response to stress and other physiological stimuli. Spectrin, actin, band 4.1, adducins, and dematin can be all phosphorylated ${ }^{[74]}$ Phosphorylation of band 4.1, combined with PKA-mediated phosphorylation of S726 and S713 in adducins results in a marked reduction in membrane stabil- ity. ${ }^{[75]}$ Both observations demonstrate, albeit by two different and distinct mechanisms, involvement of PKA in the process of ATP release by RBCs. Thus, activated PKA plays a key role in RBC plasma membrane stability via phosphorylation of the erythrocyte cytoskeleton.

We have discussed above that RBC deformability increases ATP release, and PKA phosphorylation of cytoskeletal proteins increases erythrocyte plasma membrane deformability, which leads to ATP release. Post-transcriptional modifications of RBC cytoskeletal proteins, such as phosphorylation, play a key role in regulating membrane functions, due to the lack of regulation of gene transcription in enucleate red blood cells. ${ }^{[76]}$

The RBC membrane skeleton is classically described as a structure formed by junctional complexes linked to flexible spectrin molecules. Spectrins, actin, protein 4.1, adducin, dematin, tropomyosin, and tropomodulin represent the most abundant constituents of the cytoskeleton and can all be phosphorylated by different kinases including PKA, protein kinase $\mathrm{C}$ (PKC), and casein kinases. PKA and PKC can phosphorylate adducins on Ser726 and Ser713, but only the phosphorylation by PKA reduces adducin's affinity for spectrin-F-actin complexes. ${ }^{[76]}$ Phosphorylation of band 4.1 also results in a marked decrease in membrane mechanical stability as a result of its decreased ability to bind both actin and spectrin. ${ }^{[75]}$

RBCs infected with $P$. falciparum release ATP. We have discussed above that activation of $\beta$-adrenergic receptors appears to be involved in this phenomenon, and they likely activate cAMP-dependent PKA that leads to phosphorylation of erythrocyte cytoskeletal proteins. Indeed, it has been demonstrated that $P$. falciparum infection induces post-transcriptional modification of RBC cytoskeleton ${ }^{[77]}$ and the RBC plasma membrane is extensively modified during the intracellular development of $P$. falciparum. ${ }^{[78}$ RBC serine/threonine kinases such as casein kinases, PKC, and PKA have been reported to be activated in parasitized erythrocytes. ${ }^{[76]}$ Also, activation of endogenous PKA by cAMP decreases the mechanical stability of the membrane. This effect is mediated primarily by phosphorylation of dematin, which facilitates interaction between spectrin and F-actin, and phosphorylation of dematin by PKA markedly diminishes this affinity. ${ }^{[79]}$ These findings imply that dematin also contributes to the maintenance of mechanical stability of RBC plasma membrane by facilitating spectrin-actin interactions and that phosphorylation of dematin by PKA can modulate these effects. ${ }^{[79]}$

\section{ATP release in sickle cell disease}

SCD is a hereditary disease characterized by a valine for glutamic acid substitution at position six in $\beta$-globin, which creates a hydrophobic patch in $\mathrm{HbS}$ tetramer, resulting 
in a tendency to polymerize and form long polymers that distort the shape of RBCs. ${ }^{[80]}$ Sickle RBCs are abnormally rigid, have reduced deformability, and are easily destroyed. The lifespan of RBCs of SCD patients is shortened, leading to a chronic extravascular and intravascular hemolysis and chronic hemolytic anemia. Furthermore, SCD RBCs have damaged surfaces that increase their adhesion to the vascular endothelium, a process that contributes to acute vaso-occlusion. Adenosine is the nucleotide that is highly elevated in the plasma of human patients and SCD transgenic mice. ${ }^{[72]}$ SCD patients are exposed to hypoxia, and hypoxia-induced increases in adenosine contribute to fatal complications and multiple sickling, tissue damage, pulmonary dysfunction, and priapism. ${ }^{[81]}$ Adenosine, a derivative of ATP, is not only readily released by SCD erythrocytes, but also by RBCs experiencing hypoxia, or following oxidative stress or infection by Plasmodium, as discussed below.

The ATP concentration inside RBCs is high $(\sim 5 \mathrm{mM})$ and a rise in plasma adenosine in response to hypoxia is likely due to ATP release and its conversion to adenosine. For example, ATP concentrations in the plasma of SCD transgenic mice are significantly higher compared to those observed in wild-type mice plasma. ${ }^{[72]}$ Ectonucleotidases such as CD39 on the RBC surface hydrolyze ATP and ADP to AMP ${ }^{[81]}$ Then, AMP undergoes dephosphorylation by the ectonucleotidase (CD73) that is also present on RBC surface to produce adenosine [Figure 2]. Adenosine contributes to the pathogenesis of $\mathrm{SCD}$, and in fact, it increases directly 2,3-biphosphoglycerate (2,3-BPG) levels in the RBCs,

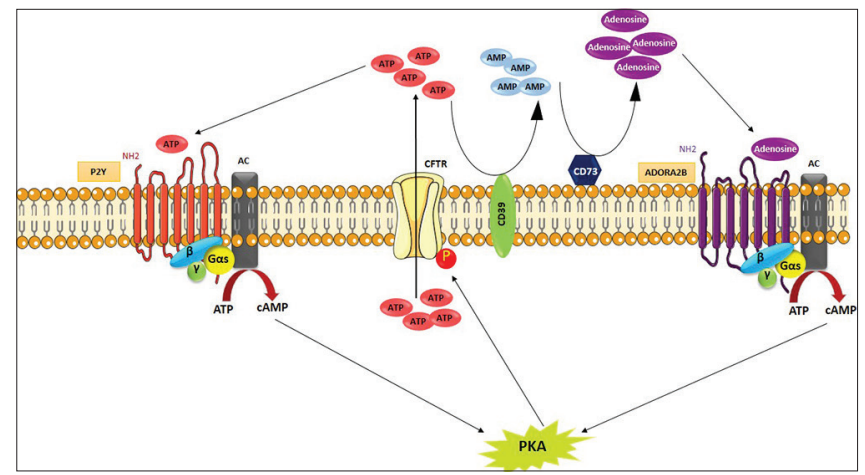

Figure 2: RBCs release ATP following P. falciparum infection. CFTR activation by PKA-mediated phosphorylation contributes to ATP release. Released ATP can signal directly via binding to RBC $\mathrm{P} 2 \mathrm{Y}$ receptors or following conversion to AMP and adenosine via the successive action of two ectonucleotidases, CD39 and CD73. ATPderived adenosine can then signal via the ADORA2B receptor. Both ADORA2B and $\mathrm{P} 2 \mathrm{Y}$ receptors are coupled to $\mathrm{G}$ proteins, and their activation ultimately leads to cAMP production and PKA activation that will play different roles in maintaining the infection process, such as CFTR phosphorylation, to sustain an ATP-release loop. Several additional players can act to regulate these pathways, such as for example, phosphodiesterases that function to degrade cAMP, thus ending the signal. which contribute to $\mathrm{O}_{2}$ being released from hemoglobin to induce sickling. ${ }^{[81]}$

It has been shown that 2,3-BPG is an RBC-specific metabolite that decreases the binding affinity of oxygen for hemoglobin, and its induction is mediated by the A2B receptor for adenosine (A2BR). ${ }^{[81]}$ Thus, excessive adenosine signaling by A2BR has a pathological role in SCD. ${ }^{[72,81]} \mathrm{Ex}-$ tracellular adenosine affects the physiological and pathological processes on target cells by signaling through different receptors including ADORA1, ADORA2A, ADORA2B, and ADORA3. All these receptors are G protein coupled and each has a distinct affinity for adenosine and a distinct cellular and tissue distribution. A2A and A2B couple to the stimulatory $\mathrm{G}$ protein, $\mathrm{Gs}$, to activate $\mathrm{AC}$ that results in increased cAMP levels. ${ }^{[82]}$

\section{Conclusions}

ATP is a nucleotide principally known as a cellular energy source, but it can also act as a potent extracellular signaling molecule. ATP can be rapidly converted to adenosine by the combined actions of the ecto-5'-nucleotidases CD39 and CD73 expressed on the RBC surface [Figure 2]. In this review, we have summarized the role of ATP as an extracellular signaling molecule in a particular cell model, $\mathrm{RBCs}$, and in the context of erythrocyte infection by malaria parasites.

Intracellular ATP concentration of RBCs can reach amounts of up to $5 \mathrm{mM}$. All this ATP present in RBCs allows them to play a role over and above just oxygen transport in blood, i.e. also as an oxygen sensor to mediate local vascular control. Indeed, following hypoxia, RBC hemoglobin undergoes deoxygenation, which initiates a cascade of events including ATP release. ATP binds to purinergic P2 subtype receptors present on the vascular endothelium and stimulates NO synthesis and its release as a potent vasodilator.

Adenosine resulting from ATP conversion is also a vasoactive mediator. It plays a crucial role in SCD; but unlike its beneficial role in healthy individuals, it plays a detrimental role in SCD patients. Increased amounts of ATP in the circulation derived from chronic sickle red cell hemolysis and tissue damage from vaso-occlusion is rapidly converted to adenosine by the combined actions of CD39 and CD73 [Figure 2].

ATP is also released by iRBCs, and this can occur in different ways. First and probably in response to hypoxia, sequestration of iRBCs causes hypoxia by obstruction of postcapillary venules. ${ }^{[83]}$ Also, iRBCs become osmotically fragile and, due to NPP, become more permeable to a wide variety of molecules. As stated above, intracellular ATP levels are altered by $\mathrm{H}_{2} \mathrm{O}_{2}$ and oxidative stress is directly correlated to ATP release. This may be another mechanism to explain 
ATP release by iRBCs. Especially, as infection with Plasmodium makes iRBCs appear older, less dense, less deformable, and more oxidatively stressed. ${ }^{[84]}$ The decreased levels of antioxidants and ATP have several consequences in malaria, such as clustering of the band-3 anion transporter, as well as exposure of neo-antigens that contribute to antigenic variation and increased iRBC adhesion, which allows them to escape destruction by spleen. $\beta$-adrenergic receptors and/or prostacyclin receptors are also implicated in ATP release by iRBCs, as they stimulate parasite entry into RBCs and augment intraerythrocytic growth. Adenosine receptors can also be implicated in addition to $\beta$-adrenergic receptors, suggesting that ATP is not the only molecule secreted upon P. falciparum infection of erythrocytes that could contribute to parasite growth.

iRBCs, damaged cells, or cells responding to a hypoxic signal can release ATP, which is an important determinant for the increased RBC invasiveness of $P$. falciparum, but ATP is probably not the only molecule involved in Plasmodium infectivity. Increased adenosine release upon P. falciparum infection may also signal via the adenosine receptor expressed on the surface of iRBCs to maintain the infection process. An involvement of $\beta$-adrenergic receptors is not to be excluded, but parasite-derived agonists responsible for their activation have not been described. However, the possibility that RBCs may function as a reservoir for noradrenaline and adrenaline had been suggested. So, adrenaline might be released in a controlled manner by iRBCs or released into plasma upon iRBC lysis. ${ }^{[85]}$

\section{Summary}

ATP release from RBCs is increased upon Plasmodium infection, where it contributes to $\mathrm{RBC}$ invasion via signaling through P2Y receptors. As RBC ATP levels are high, when the RBC plasma membrane is damaged, lysed, or traversed by Plasmodium parasites during invasion, extracellular ATP levels can increase substantially. On the RBC surface, CD39 can hydrolyze ATP and ADP to AMP and CD73 can dephosphorylate AMP to adenosine. Conditioned media from cultured iRBCs, when added to uninfected RBCs, alter their plasma membrane deformability implying that iRBCs secrete into the media a metabolite(s) that has both autocrine and in trans effects on RBC plasma membrane deformability ${ }^{[86]}$ Within a few hours of incubation of normal RBCs in spent medium from iRBC cultures, a change in the plasma membrane properties of uninfected RBCs occurred, as within $24 \mathrm{~h}$, they showed equivalent deformability changes to iRBCs ${ }^{[86]}$ Clearly, iRBCs secrete an exo-chemical compound responsible for the change in deformability of non-infected RBCs and the "bystander effect" might be due to ATP release, or its metabolites adenosine and inosine. These secreted metabolites can act in cis on iRBCs and in trans on surrounding healthy RBCs.

\section{REFERENCES}

1. Ellsworth ML, Ellis CG, Goldman D, Stephenson AH, Dietrich HH, Sprague RS. Erythrocytes: Oxygen sensors and modulators of vascular tone. Physiology (Bethesda) 2009;24:107-16.

2. Bergfeld GR, Forrester T. Release of ATP from human erythrocytes in response to a brief period of hypoxia and hypercapnia. Cardiovasc Res 1992;26:40-7.

3. Ellsworth ML, Forrester T, Ellis CG, Dietrich HH. The erythrocyte as a regulator of vascular tone. Am J Physiol 1995;269:H2155-61.

4. Sprague RS, Olearczyk JJ, Spence DM, Stephenson AH, Sprung RW, Lonigro AJ. Extracellular ATP signaling in the rabbit lung: Erythrocytes as determinants of vascular resistance. Am J Physiol Heart Circ Physiol 2003;285:H693-700.

5. Jagger JE, Bateman RM, Ellsworth ML, Ellis CG. Role of erythrocyte in regulating local $\mathrm{O} 2$ delivery mediated by hemoglobin oxygenation. Am J Physiol Heart Circ Physiol 2001;280:H2833-9.

6. Sprague RS, Ellsworth ML, Stephenson AH, Lonigro AJ. Participation of cAMP in a signal-transduction pathway relating erythrocyte deformation to ATP release. Am J Physiol Cell Physiol 2001;281:C1158-64.

7. Kirby BS, Crecelius AR, Voyles WF, Dinenno FA. Impaired skeletal muscle blood flow control with advancing age in humans: Attenuated ATP release and local vasodilation during erythrocyte deoxygenation. Circ Res 2012;111:220-30.

8. Dietrich HH, Ellsworth ML, Sprague RS, Dacey RG Jr. Red blood cell regulation of microvascular tone through adenosine triphosphate. Am J Physiol Heart Circ Physiol 2000;278:H1294-8.

9. Huang KT, Keszler A, Patel N, Patel RP, Gladwin MT, Kim-Shapiro DB, et al. The reaction between nitrite and deoxyhemoglobin. Reassessment of reaction kinetics and stoichiometry. J Biol Chem 2005;280:31126-31.

10. Gladwin MT, Shelhamer JH, Schechter AN, Pease-Fye ME, Waclawiw MA, Panza JA, et al. Role of circulating nitrite and S-nitrosohemoglobin in the regulation of regional blood flow in humans. Proc Natl Acad Sci USA 2000;97:11482-7.

11. Sprague RS, Ellsworth ML, Stephenson AH, Lonigro AJ. ATP: The red blood cell link to NO and local control of the pulmonary circulation. Am J Physiol 1996;271:H2717-22.

12. Forsyth AM, Wan J, Owrutsky PD, Abkarian M, Stone HA. Multiscale approach to link red blood cell dynamics, shear viscosity, and ATP release. Proc Natl Acad Sci USA 2011;108:10986-91.

13. Olearczyk JJ, Stephenson AH, Lonigro AJ, Sprague RS Receptor-mediated activation of the heterotrimeric G-protein Gs results in ATP release from erythrocytes. Med Sci Monit 2001;7:669-74.

14. Olearczyk JJ, Stephenson AH, Lonigro AJ, Sprague RS. Heterotrimeric $\mathrm{G}$ protein $\mathrm{Gi}$ is involved in a signal transduction pathway for ATP release from erythrocytes. Am J Physiol Heart Circ Physiol 2004;286:H940-5.

15. Sprague RS, Bowles EA, Olearczyk JJ, Stephenson AH, Lonigro AJ, The role of $\mathrm{G}$ protein beta subunits in the release of ATP from human erythrocytes. J Physiol Pharmacol 2002;53:667-74.

16. Sprague RS, Stephenson AH, Bowles EA, Stumpf MS, Lonigro AJ. Reduced expression of G (i) in erythrocytes of humans with type 2 diabetes is associated with impairment of both cAMP generation and ATP release. Diabetes 2006;55:3588-93. 
17. Sprague RS, Ellsworth ML, Stephenson AH, Kleinhenz ME, Lonigro AJ. Deformation-induced ATP release from red blood cells requires CFTR activity. Am J Physiol 1998;275:H1726-32.

18. Bayewitch ML, Avidor-Reiss T, Levy R, Pfeuffer T, Nevo I, Simonds WF, et al. Differential modulation of adenylyl cyclases I and II by various G beta subunits. J Biol Chem 1998;273:2273-6.

19. Federman AD, Conklin BR, Schrader KA, Reed RR, Bourne HR. Hormonal stimulation of adenylyl cyclase through Gi-protein beta gamma subunits. Nature 1992;356:159-61.

20. McIntire WE, MacCleery G, Garrison JC. The G protein beta subunit is a determinant in the coupling of Gs to the beta 1-adrenergic and A2a adenosine receptors. J Biol Chem 2001;276:15801-9.

21. Sunahara RK, Dessauer CW, Gilman AG. Complexity and diversity of mammalian adenylyl cyclases. Annu Rev Pharmacol Toxicol $1996 ; 36: 461-80$.

22. Tang WJ, Gilman AG. Type-specific regulation of adenylyl cyclase by G protein beta gamma subunits. Science 1991;254:1500-3.

23. Sprague R, Bowles E, Stumpf M, Ricketts G, Freidman A, Hou WH, et al. Rabbit erythrocytes possess adenylyl cyclase type II that is activated by the heterotrimeric G proteins Gs and Gi. Pharmacol Rep 2005;57 Suppl:222-8.

24. Olearczyk JJ, Stephenson AH, Lonigro AJ, Sprague RS. NO inhibits signal transduction pathway for ATP release from erythrocytes via its action on heterotrimeric G protein Gi. Am J Physiol Heart Circ Physiol 2004;287:H748-54.

25. Abbracchio MP, Burnstock G, Boeynaems JM, Barnard EA, Boyer JL, Kennedy C, et al. International Union of Pharmacology LVIII: Update on the P2Y G protein-coupled nucleotide receptors: From molecular mechanisms and pathophysiology to therapy. Pharmacol Rev 2006;58:281-341.

26. Gever JR, Cockayne DA, Dillon MP, Burnstock G, Ford AP. Pharmacology of P2X channels. Pflugers Arch 2006;452:513-37.

27. Dietrich HH, Kajita Y, Dacey RG Jr. Local and conducted vasomotor responses in isolated rat cerebral arterioles. Am J Physiol 1996;271:H1109-16.

28. Kajita Y, Dietrich HH, Dacey RG Jr. Effects of oxyhemoglobin on local and propagated vasodilatory responses induced by adenosine, adenosine diphosphate, and adenosine triphosphate in rat cerebral arterioles. J Neurosurg 1996;85:908-16.

29. Forrester T, Harper AM, MacKenzie ET. Effects of intracarotid adenosine triphosphate infusions on cerebral blood flow and metabolism in the anaesthetized baboon. J Physiol 1975;250:38-9P.

30. Sprague RS, Stephenson AH, Ellsworth ML. Red not dead: Signaling in and from erythrocytes. Trends Endocrinol Metab 2007;18:350-5.

31. Tao YP, Najafi L, Shipley S, Howlett A, Klein C. Effects of nitric oxide on adenylyl cyclase stimulation in N18TG2 neuroblastoma cells. J Pharmacol Exp Ther 1998;286:298-304.

32. Pozdnyakov N, Margulis A, Sitaramayya A. Endogenous ADP-ribosylation of a $\mathrm{G}$ (alpha i) protein in bovine ciliary body is stimulated by nitric oxide. Biochem Biophys Res Commun 1997;235:482-6.

33. Miyamoto A, Laufs U, Pardo C, Liao JK. Modulation of bradykinin receptor ligand binding affinity and its coupled G-proteins by nitric oxide. J Biol Chem 1997;272:19601-8.

34. Wan J, Ristenpart WD, Stone HA. Dynamics of shear-induced ATP release from red blood cells. Proc Natl Acad Sci U S A 2008;105:16432-7.
35. Price AK, Martin RS, Spence DM. Monitoring erythrocytes in a microchip channel that narrows uniformly: Towards an improved microfluidic-based mimic of the microcirculation. J Chromatogr A 2006;1111:220-7.

36. Moehlenbrock MJ, Price AK, Martin RS. Use of microchip-based hydrodynamic focusing to measure the deformation-induced release of ATP from erythrocytes. Analyst 2006;131:930-7.

37. Fischer DJ, Torrence NJ, Sprung RJ, Spence DM. Determination of erythrocyte deformability and its correlation to cellular ATP release using microbore tubing with diameters that approximate resistance vessels in vivo. Analyst 2003;128:1163-8.

38. Sprung R, Sprague R, Spence D. Determination of ATP release from erythrocytes using microbore tubing as a model of resistance vessels in vivo. Anal Chem 2002;74:2274-8.

39. Schafer FQ, Buettner GR. Redox environment of the cell as viewed through the redox state of the glutathione disulfide/glutathione couple. Free Radic Biol Med 2001;30:1191-212.

40. Amer J, Ghoti H, Rachmilewitz E, Koren A, Levin C, Fibach E. Red blood cells, platelets and polymorphonuclear neutrophils of patients with sickle cell disease exhibit oxidative stress that can be ameliorated by antioxidants. Br J Haematol 2006;132:108-13.

41. Lelli JL Jr, Becks LL, Dabrowska MI, Hinshaw DB. ATP converts necrosis to apoptosis in oxidant-injured endothelial cells. Free Radic Biol Med 1998;25:694-702.

42. Lee YJ, Shacter E. Oxidative stress inhibits apoptosis in human lymphoma cells. J Biol Chem 1999;274:19792-8.

43. Subasinghe W, Spence DM. Simultaneous determination of cell aging and ATP release from erythrocytes and its implications in type 2 diabetes. Anal Chim Acta 2008;618:227-33.

44. Raththagala M, Root PD, Spence DM. Dynamic monitoring of glutathione in erythrocytes, without a separation step, in the presence of an oxidant insult. Anal Chem 2006;78:8556-60.

45. de Schepper GG, van Noorden CJ, Houtkooper JM. Age-related changes of glucose-6-phosphate dehydrogenase activity in mouse oocytes. Histochem J 1987;19:467-70.

46. Rodgers GP, Lichtman HC, Sheff MF. Red blood cell glucose-6-phosphate dehydrogenase activity in aged humans. J Am Geriatr Soc 1983;31:8-11.

47. Waggiallah H, Alzohairy M. The effect of oxidative stress on human red cells glutathione peroxidase, glutathione reductase level, and prevalence of anemia among diabetics. NAm J Med Sci 2011;3:344-7.

48. Wan GH, Tsai SC, Chiu DT. Decreased blood activity of glucose-6-phosphate dehydrogenase associates with increased risk for diabetes mellitus. Endocrine 2002;19:191-5.

49. Bakhtiari N, Hosseinkhani S, Larijani B, Mohajeri-Tehrani MR, Fallah A. Red blood cell ATP/ADP and nitric oxide: The best vasodilators in diabetic patients. J Diabetes Metab Disord 2012;11:9.

50. Gallo V, Schwarzer E, Rahlfs S, Schirmer RH, van Zwieten R, Roos D, et al. Inherited glutathione reductase deficiency and Plasmodium falciparum malaria--a case study. PLoS One 2009;4:e7303.

51. Wajcman H, Galacteros F. Glucose 6-phosphate dehydrogenase deficiency: A protection against malaria and a risk for hemolytic accidents. C R Biol 2004;327:711-20.

52. Omodeo-Sale F, Motti A, Basilico N, Parapini S, Olliaro P, Taramelli D. Accelerated senescence of human erythrocytes cultured with Plasmodium falciparum. Blood 2003;102:705-11. 
53. Chen Q, Schlichtherle M, Wahlgren M. Molecular aspects of severe malaria. Clin Microbiol Rev 2000;13:439-50.

54. Sherman IW, Eda S, Winograd E. Erythrocyte aging and malaria. Cell Mol Biol (Noisy-le-grand) 2004;50:159-69.

55. Akkaya C, Shumilina E, Bobballa D, Brand VB, Mahmud H, Lang F, et al. The Plasmodium falciparum-induced anion channel of human erythrocytes is an ATP-release pathway. Pflugers Arch 2009;457:1035-47.

56. Levano-Garcia J, Dluzewski AR, Markus RP, Garcia CR. Purinergic signalling is involved in the malaria parasite Plasmodium falciparum invasion to red blood cells. Purinergic Signal 2010;6:365-72.

57. Tanneur V, Duranton C, Brand VB, Sandu CD, Akkaya C, Kasinathan RS, et al. Purinoceptors are involved in the induction of an osmolyte permeability in malaria-infected and oxidized human erythrocytes. FASEB J 2006;20:133-5.

58. Cruz LN, Wu Y, Craig AG, Garcia CR. Signal transduction in Plasmodium-Red Blood Cells interactions and in cytoadherence. An Acad Bras Cienc 2012;84:555-72.

59. Kirk K, Horner HA. Novel anion dependence of induced cation transport in malaria-infected erythrocytes. J Biol Chem 1995;270:24270-5.

60. Ginsburg H, Stein WD. The new permeability pathways induced by the malaria parasite in the membrane of the infected erythrocyte: Comparison of results using different experimental techniques. J Membr Biol 2004;197:113-34.

61. Ginsburg H, Krugliak M, Eidelman O, Cabantchik ZI. New permeability pathways induced in membranes of Plasmodium falciparum infected erythrocytes. Mol Biochem Parasitol 1983;8:177-90.

62. Saliba KJ, Horner HA, Kirk K. Transport and metabolism of the essential vitamin pantothenic acid in human erythrocytes infected with the malaria parasite Plasmodium falciparum. J Biol Chem 1998;273:10190-5.

63. Krugliak M, Zhang J, Ginsburg H. Intraerythrocytic Plasmodium falciparum utilizes only a fraction of the amino acids derived from the digestion of host cell cytosol for the biosynthesis of its proteins. Mol Biochem Parasitol 2002;119:249-56.

64. Kanaani J, Ginsburg H. Transport of lactate in Plasmodium falciparum-infected human erythrocytes. J Cell Physiol 1991;149:469-76.

65. Ayi K, Liles WC, Gros P, Kain KC. Adenosine triphosphate depletion of erythrocytes simulates the phenotype associated with pyruvate kinase deficiency and confers protection against Plasmodium falciparum in vitro. J Infect Dis 2009;200:1289-99.

66. Cruz LN, Juliano MA, Budu A, Juliano L, Holder AA, Blackman MJ, et al. Extracellular ATP triggers proteolysis and cytosolic $\mathrm{Ca}(2)(+)$ rise in Plasmodium berghei and Plasmodium yoelii malaria parasites. Malar J 2012;11:69.

67. Riordan JR, Rommens JM, Kerem B, Alon N, Rozmahel R, Grzelczak Z, et al. Identification of the cystic fibrosis gene: Cloning and characterization of complementary DNA. Science 1989;245:1066-73.

68. Childers M, Eckel G, Himmel A, Caldwell J. A new model of cystic fibrosis pathology: Lack of transport of glutathione and its thiocyanate conjugates. Med Hypotheses 2007;68:101-12.
69. Dulhanty AM, Riordan JR. Phosphorylation by cAMP-dependent protein kinase causes a conformational change in the $\mathrm{R}$ domain of the cystic fibrosis transmembrane conductance regulator. Biochemistry 1994;33:4072-9.

70. Murphy SC, Harrison T, Hamm HE, Lomasney JW, Mohandas N, Haldar K. Erythrocyte G protein as a novel target for malarial chemotherapy. PLoS Med 2006;3:e528.

71. Harrison T, Samuel BU, Akompong T, Hamm H, Mohandas N, Lomasney JW, et al. Erythrocyte G protein-coupled receptor signaling in malarial infection. Science 2003;301:1734-6.

72. Zhang Y, Dai Y, Wen J, Zhang W, Grenz A, Sun H, et al. Detrimental effects of adenosine signaling in sickle cell disease. Nat Med 2011; 17:79-86

73. Zennadi R, Whalen EJ, Soderblom EJ, Alexander SC, Thompson JW, Dubois LG, et al. Erythrocyte plasma membrane-bound ERK1/2 activation promotes ICAM-4-mediated sickle red cell adhesion to endothelium. Blood 2012;119:1217-27.

74. Mohandas N, Gallagher PG. Red cell membrane: Past, present, and future. Blood 2008;112:3939-48.

75. Manno S, Takakuwa Y, Mohandas N. Modulation of erythrocyte membrane mechanical function by protein 4.1 phosphorylation. J Biol Chem 2005;280:7581-7.

76. Pantaleo A, De Franceschi L, Ferru E, Vono R, Turrini F. Current knowledge about the functional roles of phosphorylative changes of membrane proteins in normal and diseased red cells. J Proteomics 2010;73:445-55

77. Lasonder E, Green JL, Camarda G, Talabani H, HolderAA, Langsley G, et al. The Plasmodium falciparum schizont phosphoproteome reveals extensive phosphatidylinositol and cAMP-protein kinase A signaling. J Proteome Res 2012;11:5323-37.

78. Cooke BM, Mohandas N, Coppel RL. Malaria and the red blood cell membrane. Semin Hematol 2004;41:173-88.

79. Koshino I, Mohandas N, Takakuwa Y. Identification of a novel role for dematin in regulating red cell membrane function by modulating spectrin-actin interaction. J Biol Chem 2012;287:35244-50.

80. Schnog JB, Duits AJ, Muskiet FA, ten Cate H, Rojer RA, Brandjes DP. Sickle cell disease; a general overview. Neth J Med 2004;62:364-74.

81. Zhang Y, Xia Y. Adenosine signaling in normal and sickle erythrocytes and beyond. Microbes Infect 2012;14:863-73.

82. Fredholm BB, Abbracchio MP, Burnstock G, Daly JW, Harden TK, Jacobson KA, et al. Nomenclature and classification of purinoceptors. Pharmacol Rev 1994;46:143-56.

83. Geurts N, Opdenakker G, Van den Steen PE. Matrix metalloproteinases as therapeutic targets in protozoan parasitic infections. Pharmacol Ther 2012;133:257-79.

84. Becker K, Tilley L, Vennerstrom JL, Roberts D, Rogerson S, Ginsburg H. Oxidative stress in malaria parasite-infected erythrocytes: Host-parasite interactions. Int J Parasitol 2004;34:163-89.

85. Hilario S, Saldanha C, Martins e Silva J. An in vitro study of adrenaline effect on human erythrocyte properties in both gender. Clin Hemorheol Microcirc 2003;28:89-98

86. Paul A, Pallavi R, Tatu US, Natarajan V. The bystander effect in optically trapped red blood cells due to Plasmodium falciparum infection. Trans R Soc Trop Med Hyg 2013;107:220-3. 